\section{NEW WORKWEAR BROCHURE HITS MARKETPLACE}

With 124 pages, the new Grahame Gardner brochure showcases one of their most impressive collections to date, including classic and contemporary Healthcare styles alongside Corporatewear, Scrubwear and Footwear.

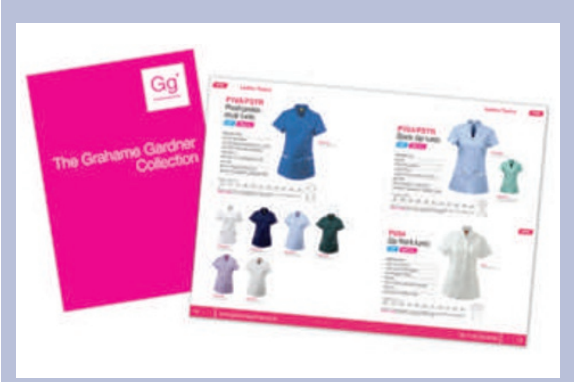

As always, Grahame Gardner have created a simple to follow brochure, so that customers can quickly locate the section that interests them. Produced in a neat handy size, this brochure features the fresh colour palette of the Grahame Gardner corporate identity and is this season's must-have essential for anyone thinking of a new identity for their dental practice.

Alongside the extensive product range, the brochure also introduces the services that demonstrate Grahame Gardner's commitment to outstanding customer care. Range Presentations and Sampling are available alongside a Measuring, Fitting and Distribution Service, developed to take the stress out of carrying out large-scale uniform ordering programmes. Their flexible manufacturing services also facilitate bespoke embroidery, printing and badges directly onto the garments, all delivered with Grahame Gardner's two-year warranty.

With improvements to their manufacturing processes and significantly increased stock facilities of the top selling lines, Grahame Gardner can now ensure that customers get their hands on their ordered products sooner than ever before.

Reader response number 53

\section{INNOVATIVE MOUTHWASH AND TOOTHPASTE}

The award-winning DECAPINOL range is an effective yet gentle approach to the treatment of gingivitis and the prevention of periodontitis. The double action of DECAPINOL works to prevent the build up of plaque and to disrupt existing plaque, simultaneously acting at different stages of the plaque formation process.

Instead of indiscriminately killing all oral bacteria like other, more harsh therapeutic agents, DECAPINOL's intelligent way of healthy plaque management inhibits the colonisation of plaque bacteria, thus helping to maintain a healthy ecological balance of the oral flora.

\section{PREVENTION BETTER THAN CURE}

DENTSPLY offer a range of products that make preventive procedures simpler for the practitioner and more comfortable for patients.

The Cavitron ultrasonic scaler system effectively removes subgingival biofilm to help towards improving periodontal health. The Cavitron can be used with a variety of inserts, including the new Cavitron THINsert for subgingival root surface debridement and the Cavitron SoftTip insert, which allows comprehensive scaling around titanium implants.

Oraqix (25/25 mg per g periodontal gel, lidocaine, prilocaine) is a non-
DECAPINOL's chlorhexidine- and SLSfree formulation, which contains the key ingredient delmopinol hydrochloride, allows patients to benefit from an effective yet safe method of preventing periodontal disease without unpleasant side-effects such as semi-permanent tooth staining. DECAPINOL Mouthwash is suitable for long-term maintenance therapy and plaque management, and can be used in conjunction with DECAPINOL Toothpaste for a complete solution to the reduction of plaque and management of gingivitis between dental visits.

Reader response number 54

injectable local anaesthetic which has been designed for use in scaling and root planing procedures. Designed with a quick onset of just 30 seconds and a relatively short duration (20 mins), it is both quick for the practitioner and short lasting for improved patient comfort. Better still, there is no needle involved.

Many hygienists like to conclude treatment by hand, and DENTSPLY's Flexichange range offers practitioners the very best in instrumentation. With soft-grip silicone handles, the range of scalers, curettes and hoes all fit comfortably in the hand, designed to minimise hand fatigue and improved grip and rotational control.

Reader response number 55

\section{A MUST-HAVE KIT FOR ALL PRACTICES}

Kerr have introduced an increased range of options to their sterile disposable examination kits and single-use periodontal instruments. The kits are ideal for high risk patients, autoclave breakdown, busy days, staff shortage and family appointments and outpatient situations.

A breakdown of the autoclave is likely to happen at some time in all dental practices and at less than $£ 2.55$ per full kit, the insurance of buying a box of kits to cover this situation is well worth the small cost to the dental practice. Using single-use kits can also save time and ease the workload on staff, who can turn their attention to improving the efficiency in other areas of their practice.

All packaging in the kit is recyclable, with some biodegradable components, and the only requirement for the sharps container is the probe. The single-use, stainless steel tipped probes and tweezers are quality instruments at affordable prices and two masks are included in the Examination Kit to encourage the continual change of masks.

Reader response number 56

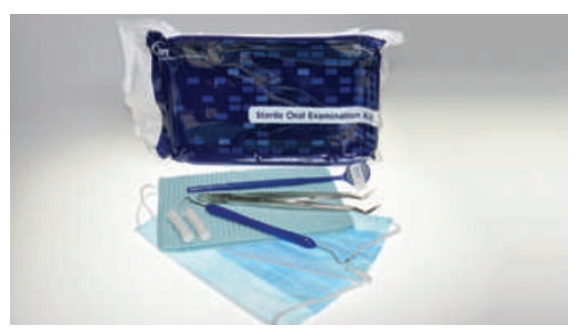

